\title{
The Temporal and Spatial Positioning Model Based on the Principle of Analemmatic Sundial in Different Parameters
}

\author{
Yu Liu ${ }^{1, a}$, Xuedong Zhu ${ }^{2, b}$ and Xuefa Xia ${ }^{3, c}$ \\ ${ }^{1}$ College of Chemical Engineering, China University of Petroleum, Beijing, 102249, China \\ ${ }^{2}$ College of Mechanical and Transportation Engineering, China University of Petroleum, Beijing, \\ 102249, China \\ ${ }^{3}$ College of Chemical Engineering, China University of Petroleum, Beijing, 102249, China \\ a752389831@qq.com, b1154114686@qq.com, '1196653289@qq.com
}

\begin{abstract}
Keywords: Shadow Trigonometric Model Analemmatic Sundial Principle $|y / x|$ Scale Model Ginput Function Dynamic Measurement Error Filter Rule
\end{abstract}

\begin{abstract}
When analyzing the data of photos and videos, it's very important to determine the shooting time and place. Usually, we can get the sun's shadow from the pictures and videos and establish different mathematical models to solve time and space positioning problems by the known parameters.

Firstly, use basic amounts to establish Analemmatic sundial model; when obtaining a series of picture messages, you can get vertex coordinate data of a fixture's sun shadow on the level ground. Then to establish $|y / x|$ scale model by Orthogonal multinomial fitting method, which can solve the latitude and longitude separately, and select $1 \%$ screening indicator as the error correction to get exact location; About shooting time, according to $|y / x|$ scale model determining the position longitude, using MATLAB to enumerate solar declination, then develop Dynamic measurement error filter rule, select the appropriate latitude and solar declination values to calculate shooting date; when getting a video recording a fixture shadow changes, use MATLAB function of Videoreader frame image, then use Rgb2gray Function convert into gray image, finally use Ginput Function reads the image coordinates. Use those to establish a comprehensive model to get the time and location of the video shoot.

Accomplish data analyzing work, which has important applications in the investigation, pointing prediction, spatial and temporal positioning and so on.

Before establishing the model, there are some assumptions are as follows:

- Assuming that the earth is a rule sphere, the entire radius are equal.

- Assuming that the sun light is parallel to the Earth, and the communication process is unhindered.

- Assuming that the number of days in a year is 365 days.

After accessing to information, we can know that the sun irradiating to the Earth's surface projected shadow, whose length is related to ascension, hour angle and solar altitude, they represent the meridional coordinate of equatorial coordinate system, the difference between the right ascension (RA) and local the sidereal time (LST), the angle formed by the Earth's equatorial plane and the line between the center of the earth and sun and the angle formed by the sun's rays and earth surface tangent [1].
\end{abstract}

\section{The Model for Analemmatic Sundial}

\subsection{Determination of the Hour Angle}

In this paper, the reference time is the time when the solar elevation angle of observation points to maximum [2], so the formula of hour angle is as follows:

$t$ - the hour angle, $T$ - the time in seconds after the reunification of the unit, $\lambda$ - the geographic longitude value. 


\subsection{Determination of the Solar Declination}

According to the law that earth move around the sun forming the declination, the formula to determine the solar declination is as follows [3]:

$N$ - the number of days since the beginning of January 1 each year, whose range is $(1,365)$.

\subsection{Determination of the Solar Elevation Angle}

Solar elevation angle is the most important factor which determining the Earth's surface to get the amount of solar thermal energy, which varies with local time and the sun's declination. Solar elevation angle is calculated as follows:

$\delta$ - the solar declination, $\varphi$ - the geographical latitude of observing location, $t$ - the hour angle.

\subsection{Determination of the Length of the Shadow}

Sun light irradiating to earth can be approximately looked as parallel light. The object itself, the shadow of the object and the light between the top of the object and the top of the shadow can form a right triangle. The length of the shadow can be determined by the trigonometric function, the formula is:

$h$ - the height of the sun, $b$ - the height of the object, $a$ - the shadow length.

Contacting the above formulas, we can get the formula which can calculate the length of shadow.

\section{The $|y / x|$ Scale Model for Point Forecast}

When doing sentinel forecasting work, we usually get some static continuous pictures about a fixture, in some cases even shooting date and fixture height are unknown. At this time, if you want to get the fixture location and date of capture, just in terms of its physical characteristics, not only it's a huge amount of work, but has low success rate. To effectively solve this problem, we establish a $|y / x|$ scale model combining dynamic measurement error analysis. According to the picture obtain fixture's shadow vertex coordinates, substitute the $|y / x|$ scale model, using MATLAB programming to determine the final fixture position coordinates and date taken. Thus greatly reduce the workload and improve the success rate.

\section{$2.1|y / x|$ Scale Model}

The moment of greatest Solar elevation angle is the local noon time, that is the shortest shadow length. If knowing noon time and comparing with a predetermined time, then know the fixture's longitude value.

For latitude, according to the principle of relativity, after converting the coordinate system, different locations for a fixture shadow trajectory is uniquely determined. Combined shadow coordinate information based on longitude and geometric dimensions, altitude value of the fixture can be calculated.

For shooting date, there is a certain relationship between the date and declination, so can use this to solve the date. After solving the shooting date, fixture location can also be obtained.

In the process of establishing the model, the fixture height is unknown. In order to avoid the position by setting the height parameter to result in uncertainty, calculate latitude and longitude innovative-separately, and apply Orthogonal-multinomial fitting method into the relationship between time points and shadows, obtain fixture's longitude value. When solving latitude, use Analemmatic sundial model to calculate time angle, and then embedded in geometry thereby obtaining fixture's latitude [4].

\subsubsection{Longitude Solving}

Through the pictures, shooting shadow's vertex coordinates $x$ and $y$ values about different moments can be obtained, using the Pythagorean theorem can get shadow length $l$, that is:

After reunification shooting time units, the $x$-coordinate for horizontal, the $y$-coordinate for vertical, as the fitting error value $\mathrm{R}^{2}$ close to 1 for the principle, select the order starting from 1 , for data points using MATLAB with Orthogonal Multinomial Fitting Method, till to $\mathrm{R}^{2}$ value nearest 1 
or equal to 1 , return to the equation with the order $m$ :

So the lowest point of the equation can be obtained, that is the minimum shadow in one day, this time is the noon time of fixture's location. Use this time minus the predetermined standard time, then expressing it in longitude, that is the fixture's location.

\subsubsection{Latitude and Date Solving}

Because the shooting date is unknown, so the value of declination is unknown, then there are two variables in $|y / x|$ scale model. But the range of latitude and declination are known, respectively $(-90,90)$ and $(-23,23)$. In view of this situation, we will model $|y / x|$ as a variable $m$, according to data, can calculate the real value of $y / x$, and based on this determine the range of $m$. Next enumerate latitude and declination, because longitude has been obtained, substitute longitude into Analemmatic sundial model, then can determine the latitude formula parameters, namely use $|y / x|$ scale model to solve the satisfying range of $m$ and the value of latitude and declination, finally obtain latitude and declination values depending on different times, analyze and select a suitable latitude and declination value as the estimated value of the location.

Latitude and declination selection rules: First, consider the actual situation that is eliminating the point at sea, and then select a value at each time point from the remaining data in the plural. Using MATLAB to solve multiple sets of latitude and declination values at different times, and then according to rules based screening program to select, then can get multiple sets of predicted values.

\subsection{Dynamic Measurement Error Filter Rule}

To further precise latitude solving, introducing dynamic measurement error analysis method, that is according to the equation, shadow length and latitude correspond with a linear relationship, so the time value and latitude should also be consistent with a linear relationship. According to the different time values to correct the calculated latitude values, can obtain difference figure and table. Select $1 \%$ as screening index for the error, to get the more precise fixture's latitude value.

\subsection{Model Application}

The shadow's coordinate data has been captured, as follows:

Table 1: A Fixture Shadow Vertex Coordinate Data Table

\begin{tabular}{|c|c|c||c|c|c||c|c|c|}
\hline Beijing Time & $X(\mathrm{~m})$ & $Y(\mathrm{~m})$ & Beijing Time & $X(\mathrm{~m})$ & $Y(\mathrm{~m})$ & Beijing Time & $X(\mathrm{~m})$ & $Y(\mathrm{~m})$ \\
\hline $14: 42$ & 1.0365 & 0.4973 & $15: 03$ & 1.2815 & 0.5368 & $15: 24$ & 1.5577 & 0.5774 \\
\hline $14: 45$ & 1.0699 & 0.5029 & $15: 06$ & 1.3189 & 0.5426 & $15: 27$ & 1.6003 & 0.5833 \\
\hline $14: 48$ & 1.1038 & 0.5085 & $15: 09$ & 1.3568 & 0.5483 & $15: 30$ & 1.6438 & 0.5892 \\
\hline $14: 51$ & 1.1383 & 0.5142 & $15: 12$ & 1.3955 & 0.5541 & $15: 33$ & 1.6882 & 0.5952 \\
\hline $14: 54$ & 1.1732 & 0.5198 & $15: 15$ & 1.4349 & 0.5598 & $15: 36$ & 1.7337 & 0.6013 \\
\hline $14: 57$ & 1.2087 & 0.5255 & $15: 18$ & 1.4751 & 0.5657 & $15: 39$ & 1.7801 & 0.6074 \\
\hline $15: 00$ & 1.2448 & 0.5311 & $15: 21$ & 1.516 & 0.5715 & $15: 42$ & 1.8277 & 0.6135 \\
\hline
\end{tabular}

According to the above calculating longitude method, use orthogonal polynomial fitting method, then give the following figure of shadow length and time:

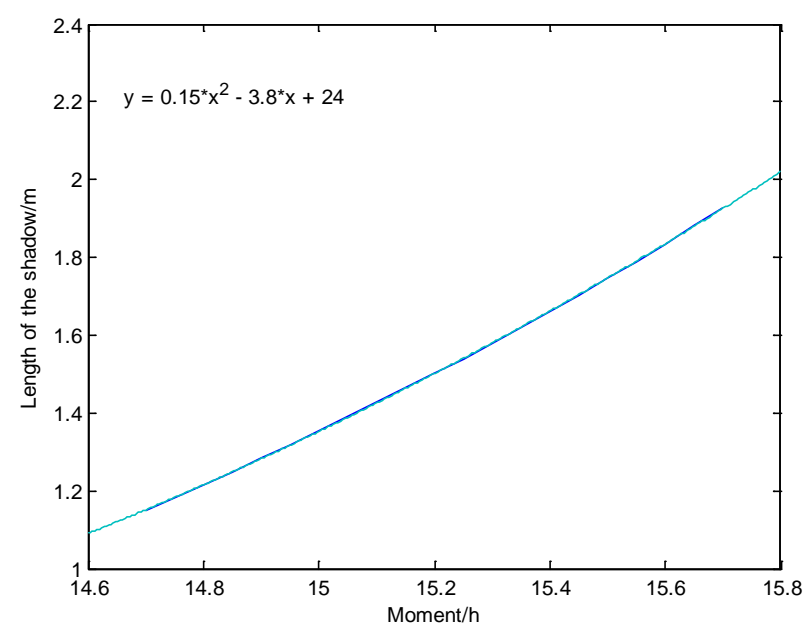

Fig.1: A Fixture Shadow and Time Fitting Figure 
According to fitting formula, you can get the abscissa of lowest point: 12.5987. Compare with the specified time, you can get the geodetic longitude value $111.0195^{\circ} \mathrm{E}$.

Next we calculate the latitude and the shooting date, in accordance with the above description, use MATLAB can get the following table:

Table 2: Multiple Sets of Latitude and Declination in Different Time Value

\begin{tabular}{|c|c|c|c|c|c|c|}
\hline Time & $14: 42$ & $14: 42$ & $14: 42$ & $14: 42$ & $14: 42$ & $14: 42$ \\
\hline$|y / x|$ & 0.250827 & 0.250827 & 0.250827 & 0.250827 & 0.250827 & 0.250827 \\
\hline$(\Phi, \delta)$ & $\begin{array}{c}(15,7) \\
(6,6)\end{array}$ & $\begin{array}{c}(25,15) \\
(13,5)\end{array}$ & $\begin{array}{c}(23,13) \\
(11,3)\end{array}$ & $\begin{array}{c}(7,12) \\
(12,16)\end{array}$ & $\begin{array}{c}(126,15) \\
(1,3)\end{array}$ & $\begin{array}{c}(35,23) \\
(16,6)\end{array}$ \\
\hline
\end{tabular}

Analyze the data and combine with the selection rules, we get $19^{\circ} \mathrm{N}$ latitude, the $10^{\circ}$ declination. Substitute into the declination equation, get $N=108$, which can get the date for April 18 .

Correspond different moments and latitude values for dynamic error measurement filter, can obtain figure as follows.

As $1 \%$ for the screening principles, accurate latitude is $19.0437^{\circ} \mathrm{N}$.

So determine the location coordinates $\left(19.0437^{\circ} \mathrm{N}, 111.0195^{\circ} \mathrm{E}\right)$, shooting date is April 18 . This data of given information are: fixture position $\left(18.3^{\circ} \mathrm{N}, 109.5^{\circ} \mathrm{E}\right)$, date April 18, 2015. The calculating values correspond with good, and close to the actual information.

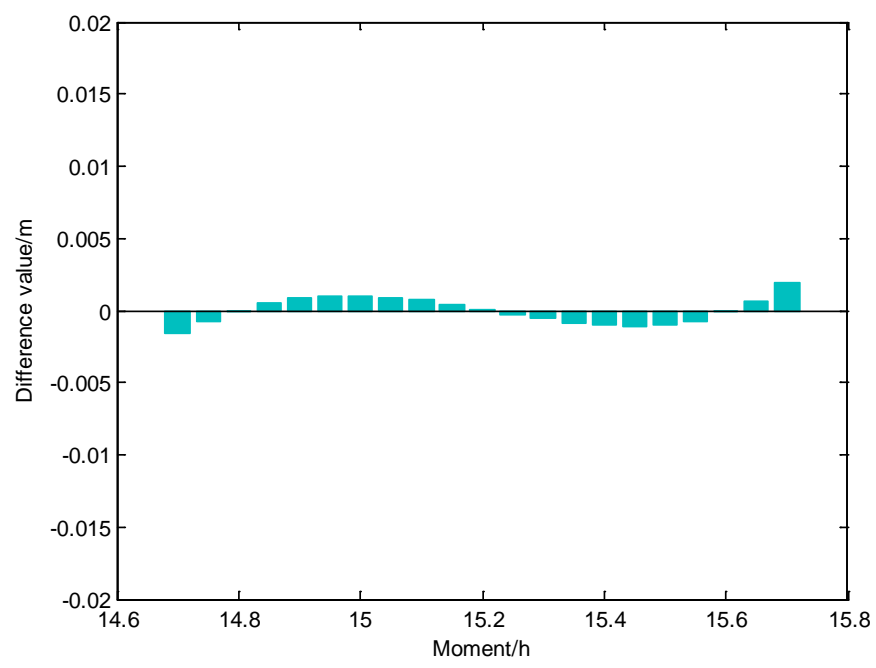

Fig.2: Time and Latitude Difference Figure

\section{The Model for Video Temporal Location}

When carrying out the investigation, unannounced visits and anti-reconnaissance work, you usually get the video information of a building and you need this information to determine the location of the shooting for expanding further track work. In order to reduce workload, improving efficiency and success rate, you should structure a video temporal positioning model.

It is difficult to determine the shadow length of a point in time from the video. Firstly, it must process the video to get the picture frame by frame. By watching the video, we can know that change took place in the length of the shadow is not obvious in a short time. If you just rely on the naked eye to observe, it must be a great error in the length of the shadow. So in order to get accurate length of the shadow, we must use a high-precision computer to process. MATLAB can do it and specific processing is as follows.

\subsection{Screen Obtaining and Gray Handling}

Frame picture is the smallest unit's single-image in screen image animation, which can reflect all the information of the video at this moment. MATLAB can process the video. Using Videoreader Function reads each of the video frames, then using the cyclic program to obtain frames that have the same time interval [5]. According to the length of the video, select the corresponding time 
interval. But the different of fixtures and shadow's color is not obvious, so it should be gray handled to highlight their main characteristics. The Ndims Function can judge whether the image is gray, Rgb2gray Function can convert a color image into a gray image. So using Ndims Function to determine whether the image is gray screen, and then using Rgb2gray Function convert a color image into a gray image. Finally, make sure that all images are completely converted into gray images.

\subsection{Determination of the Length of the Shadow}

Because the same proportional relationship between body image and the actual object, we need to determine the ratio of the fixation length and shadow length in image, and then determine the length of shadow by the ratio.

The Ginput Function can determine and give the coordinates of the crosshairs, so we can use the function to determine the coordinates $\left(x_{1}, y_{1}\right)$ of the fixture's tail or the shadow's tail (both at a point), coordinate $\left(x_{2}, y_{2}\right)$ of the fixture's top and coordinates $\left(x_{3}, y_{3}\right)$ of the shadow's top. Thus we can obtain the relationship between the length of the fixture and the length of the shadow. Similarly, we can get actual length of the shadow corresponding to all the images and make the length of shadow one by one with the time reading from the frames.

\subsection{Determination of the Data and Location of Shooting}

After solving the length of shadow, we can take the information into established model. Firstly, calculate the longitude and use MATLAB programming to select latitude and declination and determine the shooting date. Then anti-substitute the shooting date into the formula for solving the latitude to determine latitude. Finally, use dynamic measurement error screening to determine latitude and get the required information.

\section{Evaluation of the Model}

a) Because the height of fixture is unknown, we innovatively use the method which separate latitude and longitude to calculate. Avoiding deficiency and indeterminacy of results that due to the rules or parameters which set by human. Making the shadow's length and time as variable, then trying to take more values Order orthogonal polynomial curve to test which fit best. Next we make $\mathrm{R}^{2}$ as evaluation and get a perfect linear relationship. Finally use the equation to get the longitude value.

b) For video locating, we use Ginput Function in processing the video to generate the gray picture which is with the same time interval. Further, we can get the changes of the shadow length even if the date is unknown, we could combine with the previous model to obtain video shooting location and shoot time.

c) Initially, latitude value for each set of data was not the only one. In order to improve the accuracy of the results and reduce the deviation from various factors, we use dynamic measurement error screening method to analyze and exclude the error of resulting value of each set of latitude. Ultimately, determining the appropriate latitude value, and obtaining accurate result.

d) When facing a large amount of data, you can import map latitude and longitude coordinates to the software for the first time screening. Then using MATLAB to sort and classify according to the first screening to find latitude value in the middle position. In this way, it can be automated without human intervention.

e) The methods for the different types of information aim to structure the corresponding model, which can solve the special problem. Not only improving the work efficiency and reducing the workload, but also improving the accuracy and ensuring the success rate. In the investigation, anti-reconnaissance, locating prediction, temporal and spatial positioning and other fields, it has a wide range of applications.

\section{References}

[1] He Xiaolei, Yu Hejun, Li Jianying, Ding Lei, The Azimuth of the Sun and Its Solving Equations and Applications, Solar Technology, Vol. 01(2008), p. 69-72. 
[2] Qu Ming, Wang Zhengbing, Wang Dehui, Research Solar Positioning Algorithm Based on the Cross Ratio Invariance, Silicon Valley, Vol. 03(2013), p. 53-54.

[3] Information on http://www.docin.com/p-435602052.html

[4] Jiang Qiyuan, in: Mathematical Model (third edition), edited by Higher Education Press, Beijing (2003), in press.

[5] Qingyuan, in: MATLAB Advanced Applications - Graphics and Image Processing, edited by Mechanical Industry Press, Beijing (2000), in press. 